\title{
ESTIMATIVA DE ATRIBUTOS DO SOLO POR MEIO DE ESPECTRORRADIOMETRIA DIFUSA ${ }^{(1)}$
}

\author{
Everson Cezar $^{(2)}$, Marcos Rafael Nanni ${ }^{(3)}$, José Alexandre M. Demattê ${ }^{(4)}$, Marcelo Luiz \\ Chicati $^{(5)}$ \& Roney Berti de Oliveira ${ }^{(5)}$
}

\begin{abstract}
RESUMO
Diversos avanços têm sido conquistados no campo da espectrorradiometria difusa, nas últimas décadas. $\mathrm{Na}$ área agrícola, a busca por métodos de quantificação de atributos físicos e químicos do ambiente de produção, com base na reflectância do solo, tem sido pesquisada constantemente. $O$ objetivo deste trabalho foi avaliar a possibilidade de estimar os teores de silte, areia, argila, soma de bases, matéria orgânica e ferro total em amostras de solo, a partir de sua energia refletida. As amostras foram coletadas em uma área de $2.500 \mathrm{ha}$, localizada na região noroeste paranaense, composta por solos das classes Latossolo Vermelho, Nitossolo Vermelho, Argissolo Vermelho, Cambissolo Háplico e Neossolo Quartzarênico. As profundidades de amostragem foram de 0-0,2 e 0,2-0,8 m. Os pontos amostrados foram escolhidos com base em um grid de 500 × $500 \mathrm{~m}$, que recobriu toda a área de trabalho, totalizando, dessa forma, 100 pontos para o horizonte superficial e 100 pontos para o subsuperficial. As leituras espectrais foram obtidas em laboratório pelo sensor FieldSpec $3 \mathrm{JR}$. na faixa de 350 a $2.500 \mathrm{~nm}$. As curvas espectrais das amostras foram separadas em bandas e alturas, que se relacionaram com cada atributo por meio de regressão linear múltipla. A espectrorradiometria difusa evidenciou-se mais eficiente na estimação de areia, argila, soma de bases e ferro total e menos eficiente na estimação da matéria orgânica e do silte, em ambos os horizontes. Os resultados após a validação dos modelos indicam que a espectrorradiometria difusa é ferramenta promissora, que pode ser utilizada na determinação de atributos do solo.
\end{abstract}

Termos de indexação: reflectância, grid, regressão linear múltipla, predição.

\footnotetext{
(1) Parte da Tese de Doutorado desenvolvida pelo primeiro autor. Recebido para publicação em 5 de maio de 2012 e aprovado em 17 de abril de 2013.

${ }^{(2)}$ Pós-Doutorando em Agronomia, Universidade Estadual de Maringá - UEM, Av. Colombo, 5790. CEP 87020-900 Maringá (PR). E-mail: eversoncezar@yahoo.com.br

(3) Prof. Dr., PGA, UEM. E-mail: mrnanni@uem.br

(4) Prof. Dr., Departamento de Ciência do Solo, Escola Superior de Agricultura Luiz de Queiroz - ESALQ/USP. Caixa Postal 9. CEP 13418900 Piracicaba (SP). E-mail: jamdemat@usp.br

(5) Prof. Dr., Departamento de Engenharia Civil UEM. E-mail: mlchicati@yahoo.com.br; roneyberti@yahoo.com.br
} 


\title{
SUMMARY: ESTIMATION OF SOIL PROPERTIES BY MEANS OF DIFFUSE SPECTRORADIOMETRY
}

\begin{abstract}
Several advances have been made in the field of diffuse spectroradiometry in the last decades. In agriculture, the search for quantification methods of physical and chemical properties of the production environment, based on soil reflectance, has been constantly researched. The aim of this study was to evaluate the possibility of estimating the silt, sand, clay, sum of bases, total iron and organic matter content in soil samples based on the reflected energy. The samples were collected in a 2500 ha area, located in the northwestern region of Paraná, formed by Oxisol, Alfisol, Ultisol, Inceptsol, and Quartzipsamment soils, from the layers 0-0.2 and 0.2$0.8 \mathrm{~m}$. The points to be sampled were chosen based on a $500 \times 500 \mathrm{~m}$ grid, which covered the entire work area, totaling 100 samples for each layer. The spectral readings were obtained in the laboratory by the sensor FieldSpec $3 \mathrm{JR}$. in the range of 350 to $2500 \mathrm{~nm}$. The spectral curves of the samples were separated into bands and heights, which were related to each attribute by multiple linear regression. The diffuse spectroradiometry proved to be more efficient in estimating sand, clay, sum of bases, and total iron, and less efficient in the estimation of organic matter and silt, in both horizons. The results obtained after validating the models showed that diffuse spectroradiometry is a promising tool, which can be used to determine soil properties.
\end{abstract}

Index terms: reflectance, grid, multiple linear regression, prediction.

\section{INTRODUÇÃO}

Desde que o homem tem utilizado áreas específicas para o plantio de culturas, várias demandas por novas formas de análises de solo têm surgido; entretanto, essas necessitam ser mais rápidas e menos onerosas (Bouma et al., 1999; Pirie et al., 2005). No Brasil, para se realizar análises químicas de rotina são gastos, em média, U\$4,14 de reagentes por amostras de solo, além de gerar problemas de ordem ambiental, em razão dos resíduos químicos produzidos (Sousa Júnior et al., 2011).

Segundo Fiorio \& Demattê (2009), além da questão financeira e ambiental, deve-se levar em conta o tempo necessário para se obterem os resultados. Para determinar a granulometria de uma amostra pelo método de Bouyoucos (Bouyoucos, 1926), por exemplo, que foi devidamente seca e peneirada, são necessárias 48 h (Sousa Júnior et al., 2011).

Uma das alternativas que vem sendo estudada, com o intuito de contornar tais limitações, diz respeito à substituição dos atuais métodos de laboratório pela espectroscopia de reflectância difusa (DRS), que é uma técnica promissora, não destrutiva e menos onerosa, quando comparada às técnicas analíticas convencionais (Batten, 1998).

Usando o comprimento de onda do visível, infravermelho próximo e infravermelho médio, pesquisadores de várias partes do mundo têm estimado diversas propriedades, como $\mathrm{pH}$, condutividade elétrica, carbono orgânico, argila, silte, areia, soma de bases, capacidade de troca de cátions, ferro total, além de características de fertilidade como ferro $(\mathrm{Fe})$, manganês $(\mathrm{Mn})$, cobre $(\mathrm{Cu})$ e zinco $(\mathrm{Zn})$, obtendo-se acurácea variada para os resultados (Dalal \& Henry, 1986; Coleman et al., 1991; Ben-Dor \& Banin, 1995;
Demattê \& Garcia, 1999a,b; Stenberg et al., 2002; Islam et al., 2004; Sorensen \& Dalsgaard, 2005; Nanni \& Demattê, 2006a; Brown et al., 2006; Demattê et al., 2007a,b; Lee et al., 2010; Cezar et al., 2012).

De acordo com Sousa Júnior et al. (2011), alguns dos métodos estatísticos utilizados para relacionar o espectro do solo com seus atributos são: regressões linear múltipla (Demattê \& Nanni, 2003), componentes principais (Chang et al., 2001), regressão pelos mínimos quadrados parciais (Stenberg et al., 2010), regressão multivariada splines (Shepherd \& Walsh, 2002) e redes neurais (Daniel et al., 2003).

A possibilidade de quantificação de atributos do solo por meio de sua curva espectral baseia-se na intensidade da curva, assim como nas absorções de energia eletromagnética em pontos específicos ou em intervalos da curva, uma vez que diferentes quantidades de certos atributos presentes no solo podem aumentar ou diminuir a reflectância espectral em diferentes comprimentos de onda do espectro eletromagnético (Nanni, 2000).

Dessa forma, o objetivo deste trabalho foi verificar a possibilidade de estimação dos atributos silte, areia, argila, soma de bases, matéria orgânica e ferro total de uma região formada por solos derivados de basalto e arenito do Grupo Caiuá por meio de espectrorradiometria difusa.

\section{MATERIAL E MÉTODOS}

\section{Área de estudo}

A área de estudos localiza-se no município de Lobato, região noroeste paranaense, delimitada pelas coordenadas geográficas $22^{\circ} 57^{\prime} 19^{\prime \prime}$ a $22^{\circ} 52^{\prime} 59^{\prime \prime}$ 
latitude sul e $52^{\circ} 3^{\prime} 47^{\prime \prime}$ a $51^{\circ} 59^{\prime} 0.7^{\prime \prime}$ longitude oeste, apresentando dimensão de 2.500 ha (Figura 1 ).

A altitude média dessa área é de $450 \mathrm{~m}$, tendo como referência o marégrafo de Imbituba. O clima da região é classificado, segundo Köppen (1948), como Cfa, sendo subtropical úmido mesotérmico com verões quentes e geadas menos frequentes que em outras regiões do Estado, com tendências de concentração das chuvas nos meses de verão sem estação seca definida.

A escolha do local de trabalho foi realizada com base nas diferenças geológicas apresentada pela região, uma vez que se buscou avaliar uma área transicional. Situada na transição norte/noroeste do Paraná, encontra-se localizada no compartimento geomorfológico denominado Planalto de Apucarana, entre os rios Pirapó e Bandeirantes do Norte; sua geologia é composta por duas unidades dominantes constituídas por basaltos da Formação Serra Geral (JKsg), assim como arenitos friáveis do Grupo Caiuá (Kc), ambos da Era Mesozoica (ITCG, 2006). As classes de solo encontradas, de acordo com SiBCS (Embrapa, 2006), foram Latossolos Vermelhos, Nitossolos Vermelhos, Argissolos Vermelhos, Cambissolos Háplicos e Neossolos Quartzarênicos (Nanni et al., 2010).

\section{Análise laboratorial e espectral}

Foram coletadas 200 amostras de solo, 100 do horizonte superficial (0-0,2 m) e 100 do subsuperficial $(0,2-0,8 \mathrm{~m})$, com base em um grid de $500 \times 500 \mathrm{~m}$, que foi formado por 100 pontos e recobria 2.500 ha.

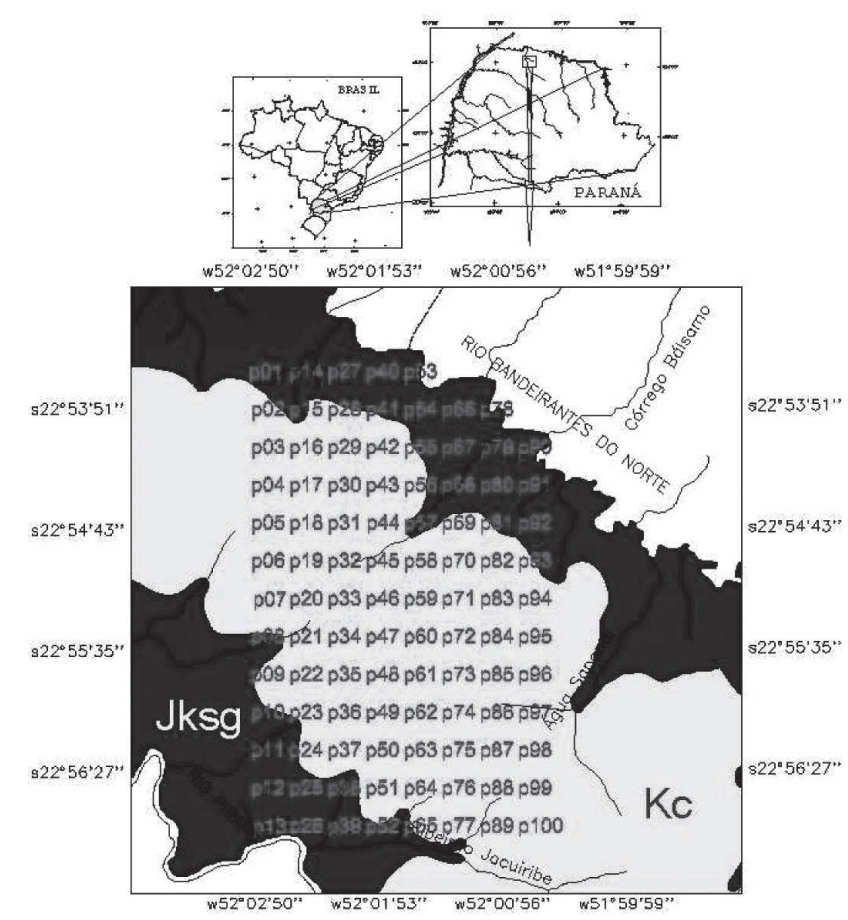

Figura 1. Área de estudo representando o grid de amostragem e a geologia local, em que JKsg: Formação Serra Geral; e Kc: Grupo Caiuá.
Desse total, $28 \%$ encontravam-se localizados em área de mata e próximos a rios, enquanto $72 \%$ encontravam-se em área com cultivo de cana-deaçúcar. Após a coleta, cada amostra de solo foi dividida em duas partes, sendo uma utilizada para caracterização física e química e a outra, para leitura espectral. As amostras submetidas às análises laboratoriais foram secas ao ar e peneiradas em malha de $2 \mathrm{~mm}$ (TFSA). Para a determinação dos teores de areia total, silte e argila, foi utilizado o método do densímetro (Kiehl, 1979). Os teores de carbono orgânico, soma de bases (SB) e $\mathrm{Fe}_{2} \mathrm{O}_{3}$ foram obtidos, conforme Embrapa (1997).

As amostras destinadas à leitura espectral foram secas em estufa à temperatura de $45^{\circ} \mathrm{C}$ por $24 \mathrm{~h}$, moídas e peneiradas (malha $2 \mathrm{~mm}$ ) para homogeneização do tamanho de partícula e redução dos efeitos da umidade e rugosidade (Epiphânio et al., 1992). Posteriormente, foram dispostas em placa de Petri com $9 \mathrm{~cm}$ de diâmetro e 1,5 cm de altura para serem submetidas à leitura espectral, em espectrorradiômetro FieldSpec $3 \mathrm{JR}$, que cobre a faixa espectral de 350 a $2.500 \mathrm{~nm}$. O equipamento foi programado para realizar 50 leituras por amostra, gerando uma curva espectral média.

Para aquisição dos dados, utilizou-se uma placapadrão branca (espectralon) com $100 \%$ de reflectância calibrada, de acordo com Labsphere Reflectance Calibration Laboratory (LRCL, 2009). O leitor de fibra óptica foi colocado em posição vertical de $8 \mathrm{~cm}$ de distância da plataforma de apoio para amostras. A área de leitura foi de aproximadamente $2 \mathrm{~cm}^{2}$. A fonte de iluminação utilizada foi uma lâmpada de $650 \mathrm{~W}$, com feixe não colimado para o plano visado, posicionada a $35 \mathrm{~cm}$ da plataforma e com um ângulo de $30^{\circ}$, em relação ao plano horizontal. As leituras espectrais foram repetidas três vezes, com deslocamento sucessivo da placa de Petri de $120^{\circ}$ no sentido horário, possibilitando total varredura da amostra. Com os dados, foi realizada a média aritmética simples das três leituras para cada amostra, de acordo com os trabalhos de Nanni \& Demattê (2006b) e Fiorio et al. (2010).

O intervalo de 350 a $2.500 \mathrm{~nm}$ das curvas espectrais foi separado em 31 bandas e 13 alturas, como descrito por Nanni et al. (2004) e Nanni \& Demattê (2006a). Nesse processo, as bandas foram escolhidas com base nas inflexões, nos intervalos entre as inflexões e nas porções côncavas presentes nas curvas de todos os solos estudados. As alturas foram escolhidas, tomando-se como base a diferença entre o maior e o menor valor de reflectância em uma banda de absorção.

\section{Análise estatística}

O estabelecimento de modelos de regressão linear múltipla para estimativa dos atributos silte, areia, argila, soma de bases, matéria orgânica e $\mathrm{Fe}_{2} \mathrm{O}_{3}$ (variáveis dependentes), com o uso das 31 bandas e 13 alturas (variáveis independentes), foi realizado no SAS 
(SAS, 2001), utilizando o procedimento de regressão stepwise, em que o sistema estabelece, passo a passo, o modelo, cujas variáveis tenham maior coeficiente de determinação $\left(R^{2}\right)$ com as variáveis independentes, as quais são selecionadas em termos de poder explicativo incremental, que podem acrescentar ao modelo de regressão (Hair et al., 1998). Além do $\mathrm{R}^{2}$, esse procedimento serviu para testar os coeficientes do modelo de regressão por meio do teste t a $5 \%$. Para melhor avaliação do modelo, na rotina do stepwise, foi inserida a opção vif collin, que determinou se as variáveis que iriam compor o mesmo apresentavam colinearidade (Souza, 1998). Aquelas variáveis que apresentaram tais características foram retiradas dos modelos.

Para comprovar a eficácia dos modelos na estimativa dos atributos em ambos os horizontes, foi realizado teste de validação, como descrito por Nanni (2000). Nessa etapa, os fatores de reflectância das bandas e alturas das amostras de solo do horizonte subsuperficial foram utilizados para testar os modelos de regressão de superfície, assim como se usaram os de reflectância das bandas e alturas das amostras de solo do horizonte superficial para testar os modelos de regressão de subsuperfície. Os valores de fator de reflectância utilizados para tal teste, embora tenham sido empregados de maneira invertida durante o processo, sempre correspondiam à mesma variável no modelo, ou seja, a banda 1 do horizonte superficial utilizou valores de fator de reflectância da banda 1 do horizonte subsuperficial e assim sucessivamente para todas as variáveis.

Essa utilização de valores invertidos se deu em razão da necessidade de utilizar amostras de solo desconhecida, ou seja, que não participaram da geração dos modelos para testar os mesmos e de avaliar se esses seriam capazes de estimar os atributos em questão. Os resultados foram confrontados com os determinados em laboratório, por meio de teste t a $5 \%$.

\section{RESULTADOS E DISCUSSÃO}

\section{Caracterização química e física dos solos}

Os resultados analíticos evidenciaram que as cinco classes de solo apresentaram teores médios de matéria orgânica acima de $20 \mathrm{~g} \mathrm{~kg}^{-1}$ no horizonte de superfície (A). A classe Latossolo Vermelho foi a que apresentou maior concentração $\left(41 \mathrm{~g} \mathrm{~kg}^{-1}\right)$, sendo seguida pelo Argissolo Vermelho ( $36 \mathrm{~g} \mathrm{~kg}^{-1}$ ), Nitossolo Vermelho (26 $\mathrm{g} \mathrm{kg}^{-1}$ ), Cambissolo Háplico e Neossolo Quartzarênico, ambos com $20 \mathrm{~g} \mathrm{~kg}^{-1}$ (Quadro 1). A soma de bases apresentou, na maioria dos casos, valores maiores nos horizontes superficiais, os quais se encontram relacionados às práticas de manejo, como adubação, calagem e gessagem, concordando com Sousa Júnior et al. (2008).

$\mathrm{O} \mathrm{Fe}_{2} \mathrm{O}_{3}$ foi encontrado em maior concentração nos horizontes subsuperficiais, assumindo valores médios superiores a $100 \mathrm{~g} \mathrm{~kg}^{-1}$, exceto para o Neossolo Quartzarênico. Os maiores valores nos horizontes de subsuperfície encontram-se relacionados à geologia regional, onde o basalto da Formação Serra Geral encontra-se recoberto pelo arenito do Grupo Caiuá, que é pobre em ferro. A análise granulométrica evidenciou que os teores de areia foram superiores aos de argila em todas as classes de solo, sendo o horizonte A o que apresentou maior concentração (Quadro 1).

\section{Estimativa de atributos do solo por meio de sua energia refletida}

A maioria dos atributos dos horizontes superficiais apresentou $R^{2}$ comum acima de 0,7 e coeficiente de variabilidade $(\mathrm{CV})$ relativamente baixo, quando comparado a trabalhos realizados por pesquisadores como Nanni et al. (2004), Nanni \& Demattê (2006a), Fiorio \& Demattê (2009), Djuuna et al. (2011). O silte e a matéria orgânica evidenciaram valores de CV mais elevados, quando comparados aos atributos argila, areia, soma de bases e ferro total (Quadro 2).

Com relação ao silte, o valor elevado do CV ocorreu em razão da diferença na concentração de óxido de ferro em cada classe de solo. Os resultados laboratoriais indicaram que o teor médio de $\mathrm{Fe}_{2} \mathrm{O}_{3}$ foi igual a $100,8 \mathrm{~g} \mathrm{~kg}^{-1}$, para o Latossolo Vermelho; $172,1 \mathrm{~g} \mathrm{~kg}^{-1}$, para o Argissolo Vermelho; 214,7 $\mathrm{g} \mathrm{kg}^{-1}$, para o Nitossolo Vermelho; $187,2 \mathrm{~g} \mathrm{~kg}^{-1}$, para o Cambissolo Háplico; e 28,2 kg-1, para o Neossolo Quartzarênico. Os teores variados de óxidos de ferro presentes nas amostras atuaram como agentes cimentantes, fazendo com que grãos de argila assumissem tamanhos e quantidades distintas de partículas de silte, influenciando na resposta espectral e na estimativa de tal atributo, concordando com Montgomery \& Baumgardner (1974), Montgomery et al. (1976), Demattê \& Garcia (1999b) e Cezar et al. (2012).

Tal afirmação é corroborada pelo resultado da análise física, em que evidenciou que o Latossolo Vermelho apresentou teor máximo de $120 \mathrm{~g} \mathrm{~kg}^{-1} \mathrm{de}$ silte, enquanto o Nitossolo Vermelho, $140 \mathrm{~g} \mathrm{~kg}^{-1}$ (dados não apresentados no quadro 1), fugindo ao padrão da área de estudo, por se tratar de local com predominância de solos mais arenosos com baixa concentração média de silte (Quadro 1).

No entanto, apesar dessa variação, o valor de $R^{2}=0,71$ para o silte foi superior ao encontrado por Nanni (2000), com valor igual a 0,27 ; por Fiorio \& Demattê (2009), com valor igual a 0,56 ; e relativamente inferior ao alcançado por Wetterlind et al. (2010), com valor de 0,73. Para a matéria orgânica, a variabilidade de cerca de $30 \%$ encontra-se relacionada à heterogeneidade do local de pesquisa, em que $28 \%$ dos pontos amostrados encontravam-se dentro de matas e próximos a rios, alcançando maiores teores de matéria orgânica (acima de $2 \%$ ), enquanto os $72 \%$ restantes encontravam-se em áreas cultivadas com cana-de-açúcar, com menores teores 
Quadro 1. Média e desvio-padrão dos atributos determinados para as classes Latossolo Vermelho (LV), Argissolo Vermelho (PV), Nitossolo Vermelho (NV), Cambissolo Háplico (CX) e Neossolo Quartzarênico (RQ)

\begin{tabular}{|c|c|c|c|c|c|c|c|c|c|c|c|c|c|c|}
\hline \multirow{2}{*}{$\begin{array}{c}\text { Classe } \\
\text { de solo } \\
\end{array}$} & \multirow{2}{*}{ Hor. ${ }^{(2)}$} & \multirow{2}{*}{$\mathbf{N}^{(3)}$} & \multirow{2}{*}{ Areia } & \multirow{2}{*}{ Silte } & \multirow{2}{*}{ Argila } & \multirow{2}{*}{$\mathbf{M O}^{(4)}$} & \multirow{2}{*}{$\mathrm{Fe}_{2} \mathrm{O}_{3}{ }^{(5)}$} & \multirow{2}{*}{$\mathbf{S B}^{(6)}$} & \multicolumn{6}{|c|}{ Desvio-padrão } \\
\hline & & & & & & & & & Areia & Silte & Argila & M.O & $\mathrm{Fe}_{2} \mathrm{O}_{3}$ & SB \\
\hline & & & & & $-\mathrm{g} \mathrm{kg}^{-1}$ & & c & $\mathrm{mol}_{\mathrm{c}} \mathrm{dm}^{-3}$ & & & & & & \\
\hline \multirow[t]{2}{*}{$\mathrm{LV}$} & A & 58 & 644 & 33 & 323 & 41 & 100,8 & 4,3 & 15,73 & 2,32 & 14,22 & 14,22 & 57,05 & 1,92 \\
\hline & Bw & 58 & 543 & 29 & 428 & 8 & 111,8 & 3,1 & 15,68 & 2,34 & 14,39 & 14,39 & 53,87 & 2,17 \\
\hline \multirow[t]{2}{*}{ PV } & $\mathrm{A}$ & 29 & 762 & 32 & 204 & 36 & 172,1 & 3,9 & 9,50 & 2,29 & 8,54 & 0,61 & 37,66 & 2,65 \\
\hline & $\mathrm{Bt}$ & 29 & 595 & 23 & 382 & 26 & 180,6 & 3,2 & 11,41 & 1,39 & 10,78 & 0,43 & 38,90 & 1,74 \\
\hline \multirow[t]{2}{*}{ NV } & $\mathrm{A}$ & 8 & 498 & 70 & 433 & 26 & 214,7 & 19,6 & 12,34 & 2,33 & 11,11 & 11,10 & 39,70 & 1,87 \\
\hline & B & 8 & 430 & 55 & 478 & 7 & 239,9 & 10,4 & 11,75 & 3,89 & 15,29 & 15,29 & 36,60 & 5,41 \\
\hline \multirow[t]{2}{*}{$\mathrm{CX}$} & A & 4 & 650 & 55 & 295 & 20 & 187,2 & 9,8 & 9,30 & 1,91 & 7,55 & 0,70 & 59,23 & 3,10 \\
\hline & $\mathrm{Bi}$ & 4 & 565 & 53 & 383 & 11 & 205,86 & 8,3 & 15,19 & 2,22 & 13,91 & 0,31 & 61,47 & 3,59 \\
\hline \multirow[t]{2}{*}{$\mathrm{RQ}$} & $\mathrm{A}$ & 1 & 830 & 30 & 140 & 20 & 28,2 & 3,3 & - & - & - & - & - & - \\
\hline & $\mathrm{C}$ & 1 & 790 & 100 & 110 & 0,4 & 39,6 & 3,5 & - & - & - & - & - & - \\
\hline
\end{tabular}

(1) Nomenclatura determinada por Embrapa (2006); ${ }^{(2)}$ Hor.: horizonte; ${ }^{(3)} \mathrm{N}$ : número de amostras; ${ }^{(4)}$ Matéria orgânica; ${ }^{(5)}$ Ferro total; $\mathrm{e}^{(6)}$ Soma de bases.

Quadro 2. Equações de regressão linear múltipla obtidas pelo uso das 31 bandas e 13 alturas, para estimação dos atributos do solo nos horizontes superficiais

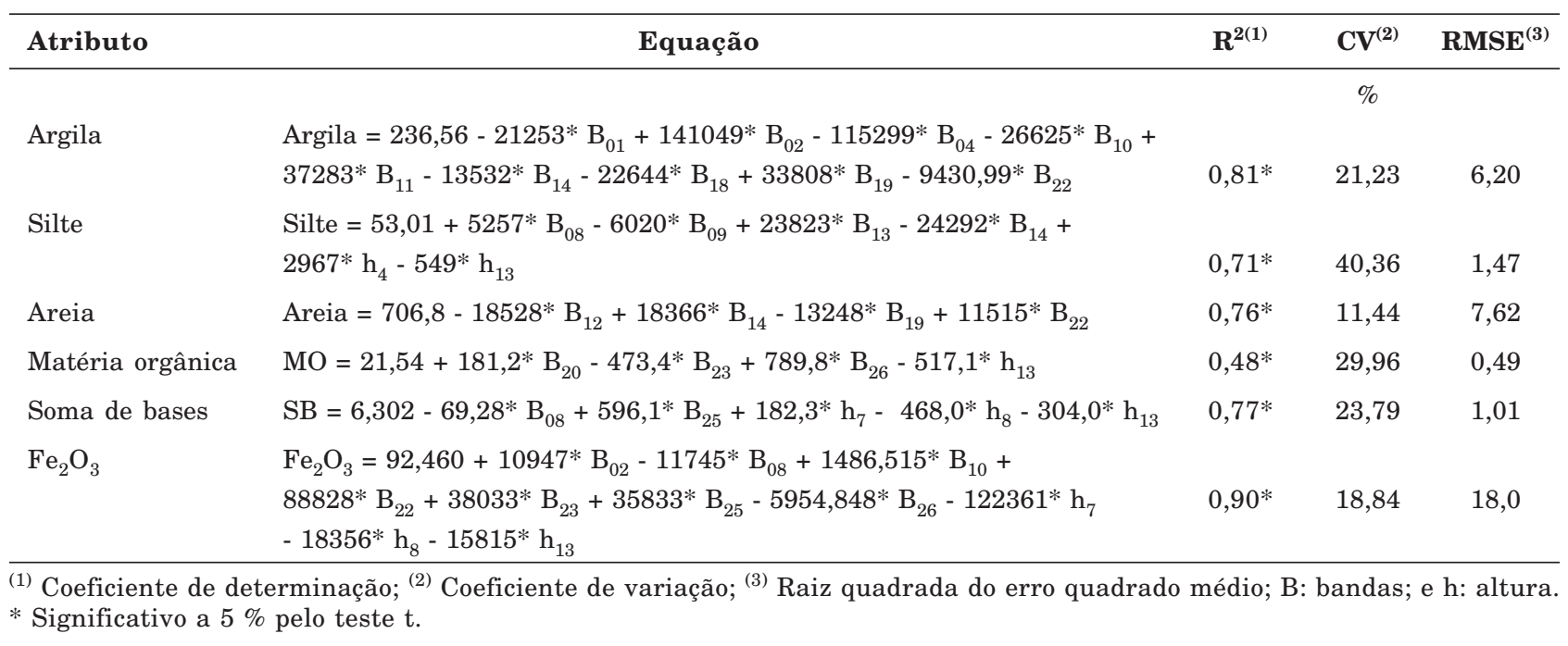

(abaixo de $2 \%$ ). O estágio de decomposição da matéria orgânica pode ter contribuído para variabilidade dos dados. Segundo Stoner (1979) e Ben-Dor \& Banin (1995), materiais mais decompostos, como aqueles presentes nas matas, apresentam menor variação espectral e baixa reflectância em relação a aqueles menos decompostos, presentes em áreas de cultivo.

Os resultados para o horizonte superficial (Quadro 2) assemelharam-se com aqueles encontrados em trabalhos realizados por Demattê \& Garcia $(1999 a, b)$ e Nanni (2000). No entanto, quando comparados aos resultados alcançados por Demattê \& Garcia (1999a,b), observou-se que as respostas foram em quase sua totalidade superiores. Entretanto, quando comparados com a pesquisa realizada por
Nanni (2000), notou-se que, para alguns atributos como argila, matéria orgânica, soma de bases e ferro total, os coeficientes de determinação obtidos foram pouco menores, significando um pior ajuste dos modelos ao conjunto de dados.

Além da comparação com o trabalho executado por Nanni (2000), os resultados alcançados para argila, silte e óxidos de ferro foram superiores a aqueles encontrados por Coleman et al. $(1991,1993)$ e Genú \& Demattê (2010), estando próximos aos obtidos por Islam et al. (2003) e Viscarra Rossel et al. (2009). Na figura 2, são apresentados os gráficos de dispersão com os valores observados e estimados, para cada atributo de solo pesquisado no horizonte superficial. 

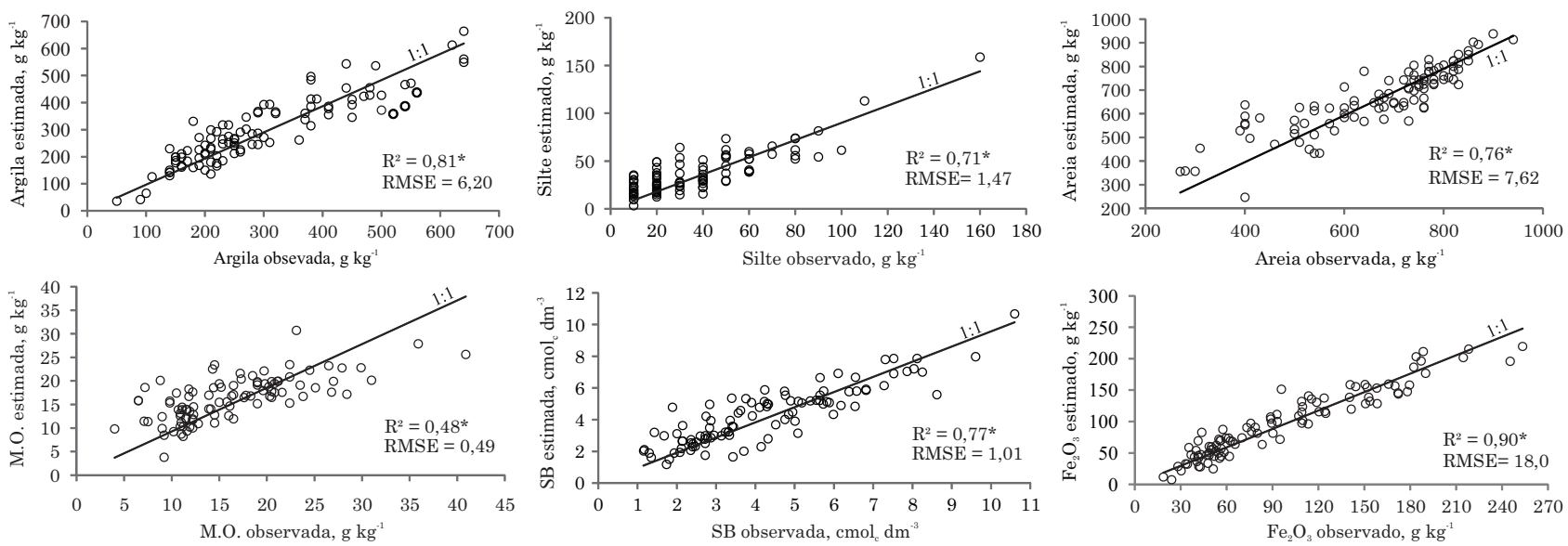

Figura 1. Gráficos de dispersão de valores observados versus estimados para argila, silte, areia, matéria orgânica (MO), soma de bases (SB) e $\mathrm{Fe}_{2} \mathrm{O}_{3}$ do horizonte superficial. RMSE: raiz quadrada do erro quadrado médio.

Como observado, os valores de argila, silte, areia, soma de bases e $\mathrm{Fe}_{2} \mathrm{O}_{3}$ obtidos por métodos analíticos e estimados pelos modelos de regressão linear múltipla apresentaram distribuição de pontos com a mesma tendência, além de baixos valores para RMSE, demonstrando a qualidade do ajustamento ao conjunto de dados.

Com relação à matéria orgânica, observou-se maior dispersão dos pontos ao redor da reta, evidenciando a dificuldade do modelo gerado em estimar esse atributo, concordando com o descrito por Sorensen \& Dalsgaard (2005) e Brown et al. (2006). $\mathrm{O} \mathrm{Fe}_{2} \mathrm{O}_{3}$ foi o atributo que apresentou o maior coeficiente de determinação $\left(R^{2}=0,90\right)$, evidenciando forte relação linear entre as variáveis. Esse resultado demonstrou que é possível estimar teores de ferro no solo com o uso da reflectância espectral, estando de acordo com Ben-Dor et al. (1999). Os resultados encontrados para as frações argila e areia apresentaram $R^{2}$ de 0,81 e 0,76 e CV de 21,23 e $11,44 \%$. Esses valores estão próximos dos alcançados por Nanni (2000), o qual obteve $\mathrm{R}^{2}$ de 0,91 , para argila, e 0,74, para areia. Da mesma maneira, assemelharam-se com os resultados encontrados por Fiorio \& Demattê (2009), os quais conseguiram valores de $\mathrm{R}^{2}$ acima de 0,8 , para ambas as frações.

Esses resultados são relativamente inferiores aos obtidos por Wetterlind \& Soderstron (2010), com R ${ }^{2}$ igual a 0,95 , para argila, e 0,91, para areia, e bem superiores aos encontrados por Genú \& Demattê (2010), com $R^{2}$ igual a 0,52, para a argila, e 0,38, para a areia, além de Sousa Junior et al. (2011), com $\mathrm{R}^{2}$ igual a 0,69 e 0,53 , respectivamente.

A soma de bases (SB) também obteve coeficiente de determinação satisfatório, sendo igual a 0,77 , com $\mathrm{CV}$ igual a $23,79 \%$; seu valor de $\mathrm{R}^{2}$ encontrou-se relativamente próximo aquele alcançado por Nanni (2000), com 0,87, e Araújo et al. (2013), com 0,69 e 0,81 , e superior ao obtido por Genú \& Demattê (2010), com 0,43 .
Os resultados para subsuperfície apresentaram a mesma tendência dos para superfície. Pôde-se observar que todos os $R^{2}$ foram superiores a 0,8 , com $C V$ relativamente baixo (Quadro 3). No entanto, tal fato não foi constatado novamente para a matéria orgânica e a fração silte, que apresentaram $R^{2}$ igual a 0,54 e 0,62 e CV igual a 24,20 e $49,66 \%$, respectivamente.

A maior capacidade de estimação dos atributos areia e SB no horizonte subsuperficial encontrou-se ligada ao fato de que nessa porção do perfil $(0,80-1,0 \mathrm{~m})$ a quantidade de matéria orgânica detectada foi em média menor que $20 \mathrm{~g} \mathrm{~kg}^{-1}$ (Quadro 1) para todas as classes de solo estudadas. Dessa forma, outros constituintes passaram a ter maior influência sobre a curva espectral do que a matéria orgânica, concordando com o descrito por Baumgardner et al. (1970) e Dalmolin et al. (2005).

$\mathrm{O}$ modelo obtido para o $\mathrm{Fe}_{2} \mathrm{O}_{3}$ apresentou menor capacidade de predição, quando comparado ao do horizonte superficial. Por meio da equação, pode-se observar que o número de variáveis correlacionadas com o atributo ferro foi maior no horizonte superficial (10 variáveis), quando comparado ao do horizonte subsuperficial (seis variáveis), evidenciando que, embora o horizonte superficial apresentasse maior teor de matéria orgânica, essa não foi capaz de mascarar as bandas características da presença de ferro, discordando de Galvão \& Vitorello (1998).

Portanto, é provável que os resultados estejam relacionados à forma do ferro e não à quantidade de matéria orgânica. Segundo Demattê \& Garcia (1999b) e Demattê et al. (2003), óxidos de ferro com elevada cristalinidade, além de reduzirem a intensidade da curva em todo seu espectro-óptico, apresentam bandas específicas de absorção, enquanto os não cristalinos influenciam somente na intensidade da curva, porém de forma mais intensa. Considerando que todas as classes de solo investigadas apresentaram teores de ferro extraível pelo ataque sulfúrico maiores nos 
horizontes subsuperficiais, em relação aos superficiais (Quadro 1), era de se esperar que houvesse maior absorção de energia nessa porção do perfil, o que de fato ocorreu. Na figura 3, estão apresentados os valores observados e estimados para os atributos dos horizontes subsuperficiais.

Mais uma vez a matéria orgânica demonstrou ser um atributo difícil de estimar, embora o resultado desta pesquisa tenha sido melhor do que aquele alcançado por Nanni (2000), Chicati (2011) e Sousa Júnior et al. (2011), os quais ajustaram modelos com $\mathrm{R}^{2}$ igual a $0,32,0,24$ e 0,27 , respectivamente. A dificuldade em se estimar esse atributo encontra-se relacionada com os seus constituintes, que apresentam reflectância total baixa, em razão da capacidade que esses possuem em absorverem a energia eletromagnética (Obukhov \& Orlov, 1964; Flaig et al., 1975).
A areia foi o atributo com melhor capacidade de ser estimado, apresentando $\mathrm{R}^{2}$ igual a $0,90, \mathrm{CV}$ igual a $9,02 \%$ e RMSE igual a 4,97. Isso se deve ao fato de que como nessa porção do perfil o teor de matéria orgânica ficou abaixo de $20 \mathrm{~g} \mathrm{~kg}^{-1}$, houve maior espalhamento da luz em razão da presença do mineral quartzo, mascarando a absorção da matéria orgânica, estando de acordo com o preconizado por Stenberg et al. (2002).

O teste t aplicado nos resultados, durante a validação, evidenciou que a média estimada para areia, argila, soma de bases e $\mathrm{Fe}_{2} \mathrm{O}_{3}$, pelos modelos de superfície, não diferiu estatisticamente daquela determinada em laboratório por métodos analíticos. No entanto, as médias para fração silte e matéria orgânica diferiram-se estatisticamente, quando comparadas com os resultados de laboratório (Quadro 4).

Quadro 3. Equações de regressão linear múltipla obtidas pelo uso das 31 bandas e 13 alturas, para estimação dos atributos do solo nos horizontes subsuperficiais

\begin{tabular}{|c|c|c|c|c|}
\hline Atributo & Equação & $\mathbf{R}^{2(\mathbf{1})}$ & $\mathbf{C V}^{(2)}$ & $\mathbf{R M S E}^{(3)}$ \\
\hline & & & $\%$ & \\
\hline Argila & $\begin{array}{l}\text { Argila }=454,11+15835^{*} \mathrm{~B}_{08}-32470 * \mathrm{~B}_{09}+27326^{*} \mathrm{~B}_{11}-12528^{*} \mathrm{~B}_{16}+ \\
30544^{*} \mathrm{~B}_{20}-7704,2^{*} \mathrm{~B}_{28}-20544^{*} \mathrm{~h}_{5}\end{array}$ & $0,80^{*}$ & 15,23 & 6,32 \\
\hline Silte & Silte $=16,58+14479 * \mathrm{~B}_{18}-12360 * \mathrm{~B}_{19}-1982,2 * \mathrm{~h}_{6}$ & $0,62^{*}$ & 49,66 & 1,50 \\
\hline Areia & $\begin{array}{l}\text { Areia }=530,83-16009 * \mathrm{~B}_{08}+26635 * \mathrm{~B}_{09}-22919 * \mathrm{~B}_{11}+32825 * \mathrm{~B}_{16}+ \\
42077 * \mathrm{~B}_{18}-43720 * \mathrm{~B}_{19}-15050 * \mathrm{~B}_{27}+67430 * \mathrm{~B}_{28}-24050 * \mathrm{~h}_{4}- \\
82977 * \mathrm{~h}_{11}+35491 * \mathrm{~h}_{13}\end{array}$ & $0,90 *$ & 9,02 & 4,97 \\
\hline Matéria orgânica & $\mathrm{MO}=15,39-103,5^{*} \mathrm{~B}_{11}+374,1^{*} \mathrm{~B}_{26}-319,9^{*} \mathrm{~B}_{30}$ & $0,54^{*}$ & 24,20 & 0,18 \\
\hline Soma de bases & $\begin{array}{l}\mathrm{SB}=1,186+72,35^{*} \mathrm{~B}_{10}-169,50 * \mathrm{~B}_{15}+956,40 * \mathrm{~B}_{17}+845,0^{*} \mathrm{~B}_{18}- \\
193,8^{*} \mathrm{~B}_{23}+424,90 * \mathrm{~B}_{25}-1590,0^{*} \mathrm{~h}_{5}-324,60 * \mathrm{~h}_{13}\end{array}$ & $0,82^{*}$ & 29,34 & 0,97 \\
\hline $\mathrm{Fe}_{2} \mathrm{O}_{3}$ & $\begin{array}{l}\mathrm{Fe}_{2} \mathrm{O}_{3}=125,3-7879 * \mathrm{~B}_{10}+10354 * \mathrm{~B}_{11}-4711^{*} \mathrm{~B}_{16}+3526 * \mathrm{~B}_{19}- \\
16670^{*} \mathrm{~B}_{28}+15235^{*} \mathrm{~h}_{10}\end{array}$ & $0,88^{*}$ & 16,87 & 18,74 \\
\hline
\end{tabular}

(1) Coeficiente de determinação; ${ }^{(2)}$ Coeficiente de variação; ${ }^{(3)}$ Raiz quadrada do erro quadrado médio; B: bandas; e h: altura.

* Significativo a $5 \%$ pelo teste t.
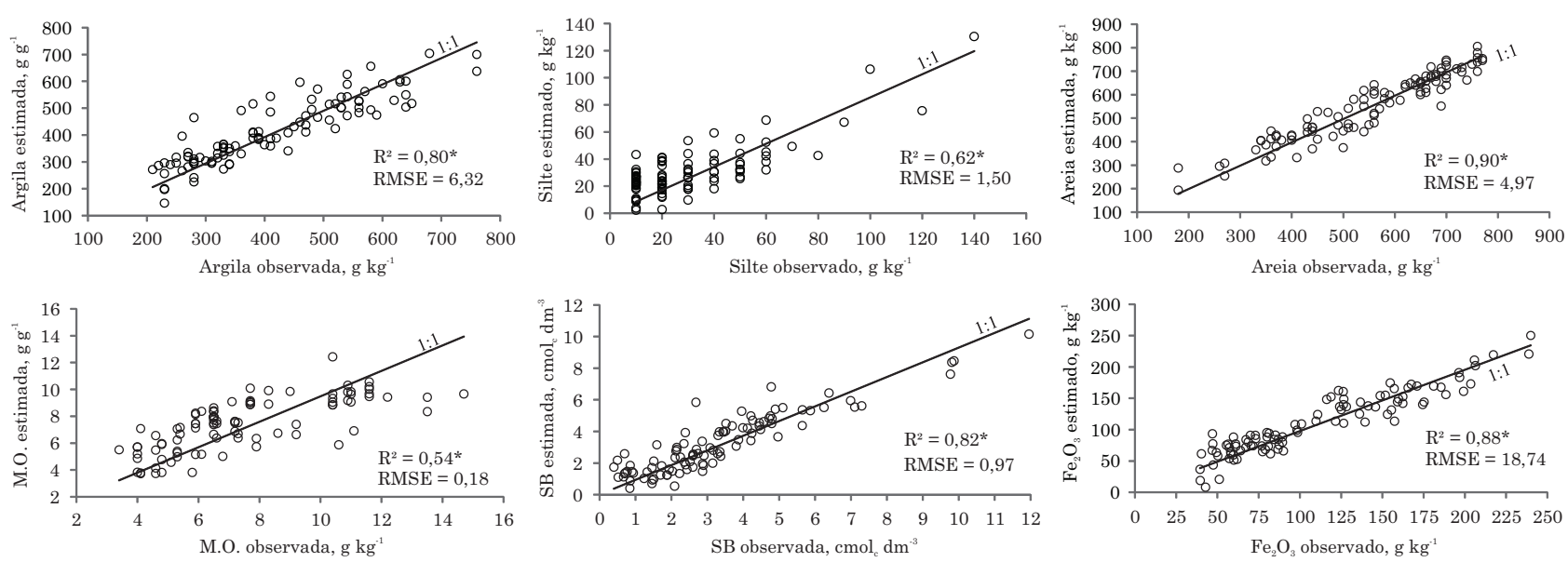

Figura 2. Gráficos de dispersão de valores observados versus estimados para argila, silte, areia, matéria orgânica, soma de bases e ferro total do horizonte subsuperficial. RMSE: raiz quadrada do erro quadrado médio. 
Os resultados obtidos mediante o uso dos modelos de subsuperficie evidenciaram que somente a fração areia não apresentou diferença significativa, quando utilizadas as bandas e alturas advindas de amostras de superfície. Apesar de a concentração média de matéria orgânica ser acima de $20 \mathrm{~g} \mathrm{~kg}^{-1}$ para as amostras utilizadas no teste do modelo, esse teste demonstrou ser eficiente e capaz de estimar o atributo areia, de tal forma que os valores estimados e observados não apresentassem diferenças significativas a $5 \%$. Esse resultado encontra-se atrelado ao descrito por Stenberg et al. (2002) e Demattê \& Nanni (2003). Ressalta-se que $29 \%$ das amostras de solo foram representadas pela classe Argissolo Vermelho, que apresenta gradiente textural com maior concentração de areia no horizonte superficial (Embrapa, 2006). As amostras desse horizonte apresentaram forte efeito do espalhamento da luz, suprimindo o intenso efeito da matéria orgãnica, gerando melhores respostas espectrais, que possibilitaram que o modelo fosse capaz de estimar adequadamente 0 atributo areia.

No entanto, a média dos atributos silte, argila, matéria orgânica, soma de bases e $\mathrm{Fe}_{2} \mathrm{O}_{3}$ apresentou diferença significativa a $5 \%$, quando comparada pelo "teste t" (Quadro 4).

A existência de diferença entre os valores observados e estimados provavelmente encontra-se relacionada à interação energia-matéria. Essa afirmação deve-se ao fato de que como a resposta espectral do solo é resultante da interação entre a energia radiante e as propriedades do mesmo, equações de regressão linear múltipla poderiam ser utilizadas somente para amostras de solo advindas de horizontes semelhantes aos utilizados no estabelecimento da equação, concordando com Nanni (2000).

Portanto, neste estudo, como se tratou de uma transição arenito/basalto, formando diversas topossequências com diferentes classes de solo, os modelos foram gerados de acordo com as características espectrais de seus respectivos atributos. Dessa forma, as equações ajustadas para o horizonte superficial foram equações que contemplaram o forte efeito da matéria orgânica, uma vez que essa se encontrava em maior concentração nessa porção do perfil. Entretanto, as equações ajustadas para o horizonte subsuperficial contemplaram o efeito de outros atributos, principalmente a areia, que possui forte reflectância (Stenberg et al., 2002; Resende et al., 2005).

A variação na concentração e mineralogia dos atributos entre os horizontes influenciou na resposta espectral das amostras, que, por sua vez, influenciaram na geração dos modelos. Quando os modelos ajustados com as amostras provenientes do horizonte superficial foram testados pelas bandas e alturas obtidas das amostras advindas do horizonte subsuperfical, erros de subestimação ou superestimação foram gerados, prejudicando a melhor estimação dos atributos. O mesmo ocorreu quando os modelos ajustados com as amostras de subsuperficie foram testados pelas bandas e alturas obtidas das amostras de superfície.

\section{CONCLUSÕES}

1. A espectrorradiometria difusa evidenciou ser capaz de estimar os atributos areia, argila, soma de bases e, principalmente, $\mathrm{Fe}_{2} \mathrm{O}_{3}$, da área de transição arenito/basalto do noroeste do Estado do Paraná sendo alcançados melhores resultados para os horizontes superficiais.

2. A matéria orgânica e o silte apresentaram menor capacidade de estimativa com o uso da espectrorradiometria difusa, obtendo, respectivamente, $\mathrm{R}^{2}$ igual a 0,71 e 0,48, para o horizonte superficial, e 0,62 e 0,54 , para o horizonte subsuperficial, demonstrando a dificuldade em estimar tais atributos a partir do método empregado.

Quadro 4. Comparação de médias (n=100) determinadas em laboratório por métodos analíticos e estimadas pela equação de regressão linear múltipla para os horizontes superficial e subsuperficial, utilizando o "teste t"

\begin{tabular}{|c|c|c|c|c|c|c|}
\hline & Areia & Silte & Argila & MO $^{(3)}$ & $\mathbf{S B}^{(4)}$ & $\mathrm{Fe}_{2} \mathrm{O}_{3}^{(5)}$ \\
\hline & \multicolumn{4}{|c|}{$\mathrm{g} \mathrm{kg}^{-1}$} & \multirow[t]{2}{*}{$\mathrm{cmol}_{\mathrm{c}} \mathrm{dm}^{-3}$} & \multirow[t]{2}{*}{$\mathrm{g} \mathrm{kg}^{-1}$} \\
\hline & & & Horizo & rficial & & \\
\hline Observado $^{(1)}$ & $551,50 \mathrm{a}$ & $33,20 \mathrm{a}$ & $415,30 \mathrm{a}$ & $7,75 \mathrm{a}$ & $3,49 \mathrm{a}$ & 111,92 \\
\hline \multirow[t]{2}{*}{ Estimado $^{(2)}$} & 546,88 a & $42,82 \mathrm{~b}$ & $410,30 \mathrm{a}$ & $9,40 \mathrm{~b}$ & $3,59 \mathrm{a}$ & 116,15 \\
\hline & \multicolumn{6}{|c|}{ Horizonte subsuperficial } \\
\hline Observado $^{(1)}$ & $666,80 \mathrm{a}$ & $38,2 \mathrm{a}$ & $295,00 \mathrm{a}$ & $16,58 \mathrm{a}$ & $4,20 \mathrm{a}$ & $95,62 \mathrm{a}$ \\
\hline Estimado $^{(2)}$ & $655,37 \mathrm{a}$ & $24,95 \mathrm{~b}$ & $319,68 \mathrm{~b}$ & $9,40 \mathrm{~b}$ & $3,07 \mathrm{~b}$ & $115,41 \mathrm{k}$ \\
\hline
\end{tabular}

(1) Valor médio dos atributos determinados em laboratório; ${ }^{(2)}$ Valor médio dos atributos determinados pelas equações de regressão múltipla; ${ }^{(3)}$ Matéria orgânica; ${ }^{(4)}$ Soma de bases; ${ }^{(5)}$ Ferro total. Médias seguidas por letras diferentes nas colunas diferenciamse pelo teste t a $5 \%$; e n: número de indivíduos empregados na obtenção da média para cada atributo. 
3. O emprego da resposta espectral do solo na estimativa de atributos físicos e químicos foi promissor, indicando que essa técnica é ferramenta valiosa na predição desses, embora o estudo tenha sido realizado em uma área de transição arenito/basalto.

\section{AGRADECIMENTOS}

Ao CNPq, pela concessão da bolsa de doutorado ao primeiro autor (Processo n ${ }^{\circ}$ 141874/2009-0), e da bolsa de produtividade ao segundo autor (Processo $\mathrm{n}^{\circ}$ 303989/2009-2).

\section{LITERATURA CITADA}

ARAÚJO, S.R.; DEMATTÊ, J.A.M. \& BELLINASO, H. Analysing the effects of applying agricultural lime to soils by VNIR spectral sensing: A quantitative and quick method. Inter. J. Remote Sens., 34:4570-4584, 2013.

BAUMGARDNER, M.F.; KRISTOF, S.; JOHANNSEN, C.J. \& ZACHARY, A. Effects of organic matter on the multispectral properties of soils. Proc. Ind. Acad. Sci., 79:413-422, 1970.

BATTEN, G.D. Plant analysis using near infrared reflectance spectroscopy: The potential and the limitations. Aust. J. Exp. Agric., 38:697-706, 1998.

BEN-DOR, E. \& BANIN, A. Near-infrared analysis as a rapid method to simultaneously evaluate several soil properties. Soil Sci. Soc. Am. J., 59:364-372, 1995.

BEN-DOR, E.; IRONS, J.H. \& EPEMA, G.F. Soil reflectance. In: RENCZ, A.N., ed. Remote sensing for the earth sciences. New York, J. Wiley \& Sons, 1999. p.111-188.

BOUMA, J.; STOORVOGEL, J.; ALPHEN, B.J. \& BOOLTING, H.W.G. Pedology, precision agriculture, and the changing paradigm of agricultural research. Soil Sci. Soc. Am. J., 63:1763-1768, 1999.

BOUYOUCOS, G.J. Estimation of the colloidal material in soils. Science, 64:362, 1926.

BROWN, D.J.; SHEPHERD, K.D.; WALSH, M.G.; MAYS, M.D. \& REINSCH, T.G. Global soil characterization with VNIR diffuse reflectance spectroscopy. Geoderna, 132:273-290, 2006.

CEZAR, E.; NANNI, M.R. CHICAT, M.L.; SOUZA JÚNIOR, I.G. \& COSTA, A.C.S. Avaliação e quantificação das frações silte, areia e argila por meio de suas respectivas reflectâncias. R. Bras. Ci. Solo, 36:1157-1165, 2012.

COLEMAN, T.L.; AGBU, P.A.; MONTGOMERY, O.L.; GAO, T. \& PRASAD, S. Spectral band selection for quantifying selected properties in highly weathered soils. Soil Sci., 151:355-361, 1991.

COLEMAN, T.L.; AGBU, P.A. \& MONTGOMERY, O.L. Spectral differentiation of surface soils and soil properties: Is it possible from space platforms? Soil Sci., 155:283-293, 1993.
CHANG, C.; LAIRD, D.A.; MAUSBACH, M.J. \& HURBURGH JUNIOR, C.R. Near-infrared reflectance spectroscopy principal components regression analyses of soil properties. Soil Sci. Soc. Am. J., 65:480-490, 2001.

CHICATI, M.L. Avaliação espectral de solos em áreas alagáveis do noroeste do Paraná. Maringá, Universidade Estadual de Maringá, 2011. 185p. (Tese de Doutorado)

DALMOLIN, R.S.D.; GONÇALVES, C.N.; KLAMT, E. \& DICK, D.P. Relação entre os constituintes do solo e seu comportamento espectral. Ci. Rural, 35:481-489, 2005.

DALAL, R.C. \& HENRY, R.J. Simultaneous determination of measure, organic carbon, and total nitrogen by near infrared spectrophotometry. Soil Sci. Soc. Am. J., 50:120$123,1986$.

DANIEL, K.W.; TRIPATHI, N.K. \& HONDA, K. Artificial neural network analysis of laboratory and in situ spectra for the estimation of macronutrients in soils of Lop Buri (Thailand). Aust. J. Soil Res, 41:47-59, 2003.

DEMATTÊ, J.A.M. \& GARCIA, G.J. Alteration of soil properties through a weathering sequence as evaluated by spectral reflectance. Soil Sci. Soc. Am. J., 63:327-342, 1999a.

DEMATTÊ, J.A.M. \& GARCIA, G.J. Avaliação de atributos de Latossolo Bruno e de terra Bruna Estruturada na região de Guarapuava, Paraná, por meio de sua energia refletida. R. Bras. Ci. Solo, 23:343-355, 1999b.

DEMATTÊ, J.A.M.; EPIPHANIO, J.C.N. \& FORMAGGIO, A.R. Influência da matéria orgânica e de formas de ferro na reflectância de solos tropicais. Bragantia, 62:451-464, 2003.

DEMATTÊ, J.A.M. \& NANNI, M.R. Weathering sequence of soils developed from basalt as evaluated by laboratory (IRIS), airborne (AVIRIS) and orbital (TM) sensors. Inter. J. Remote Sens., 24:4715-4738, 2003.

DEMATTÊ, J.A.M.; NANNI, M.R.; FORMAGGIO, A.R. \& EPIPHANIO, J.C.N. Spectral reflectance for the mineralogical evaluation of Brazilian low clay activity soils. J. Remote Sens., 28:4537-4559, 2007a.

DEMATTÊ, J.A.M.; GALDOS, M.V.; GUIMARÃES, R.V.; GENÚ, A.M.; NANNI, M.R. \& JÚNIOR, J.Z. Quantification of tropical soil attributes from ETM+/ LANDSAT 7 data. J. Remote Sens., 28:3813-3829, 2007b.

DJUUNA, I.A.F.; ABBOOTT, L. \& RUSSELL, C. Determination and prediction of some soil properties using partial least square (PLS) calibration and mid-infrared (MIR) spectroscopy analysis. J. Trop. Soils, 16:93-98, 2011.

EMPRESA BRASILEIRA DE PESQUISA AGROPECUÁRIA EMBRAPA. Manual de métodos de análises de solo. 2.ed. Rio de Janeiro, 1997. 212p.

EMPRESA BRASILEIRA DE PESQUISA AGROPECUÁRIA EMBRAPA. Centro Nacional de Pesquisa do Solo. Sistema brasileiro de classificação de solos. 2.ed. Brasília, 2006. $412 \mathrm{p}$.

EPIPHÂNIO, J.C.N.; FORMAGIO, A.R.; VALERIANO, M.M. \& OLIVEIRA, J.B. Comportamento espectral dos solos do estado de São Paulo. São José dos campos, INPE, 1992. 132p. 
FIORIO, P.R. \& DEMATTÊ, J.A.M. Orbital and laboratory spectral data to optimize soil analysis. Sci. Agríc., 66:250$275,2009$.

FIORIO, P.R.; DEMATTÊ, J.A.M.; NANNI，M.R. \& FORMAGGIO, A.R. Diferenciação espectral de solo utilizando dados obtidos em laboratórios e por sensor orbital. Bragantia, 69:453-466, 2010.

FLAIG, W.; BEUTELSPACHER, H. \& RIETZ, E. Chemical composition and physical properties of humic substances. In: GIESEKING, J.E., ed. Soil components. New York, 1975. p.1-211.

GALVÃO, L.S. \& VITORELLO, I. Role organic matter in obliterating the effects of iron on spectral reflectance and colour of Brazilian tropical soils. Inter. J. Remote Sens., 19:1969-1979, 1998.

GENÚ, A.M. \& DEMATTÊ, J.A.M. Informações espectroeletromagnéticas e topográficas na determinação de teores de componentes do solo. Bragantia, 69:157-164, 2010.

HAIR, J.F.; TATHAM, R.L.; ANDERSON, R.E. \& BLACK, W. Multivariate data analyses. 5.ed. Porto Alegre, Artmed, 1998. $597 \mathrm{p}$.

INSTITUTO DE TERRAS, CARTOGRAFIA E GEOCIÊNCIAS - ITCG. Carta de descrição das unidades litoestratigráficas. Curitiba, 2006. Folha SF-22-Y-B (Presidente Prudente), Escala 1: 250.000.

ISLAM, K.; SINGH, B. \& McBRATNEY, A. Simultaneous estimation of several soils properties by ultra-violet, visible and near-infrared reflectance spectroscopy. Aust. J. Soil Res., 41:1101-1114, 2003.

ISLAM, K.; SINGH, B.; SCHWENKE, G. \& McBRATNEY, A. Evaluation of vertosol soil fertility using ultra-violet, visible and near-infrared reflectance spectroscopy. In: SUPERSOIL, 3. 2004, Sydney. Proceedings... Sydney, University of Sydney, 2004. CD-ROM

KIEHL, E.J. Manual de edafologia - Relações solo-planta. 2.ed. São Paulo, Agronômica Ceres, 1979. 266p.

KÖPPEN, W.M. Climatologia: Con un studio de los climas de la terra. México, Fondo de Cultura Económica, 1948. 478p.

LABSPHERE REFLECTANCE CALIBRATION LABORATORY - LRCL. Spectral reflectance target calibrated from $0.25-2.5 \mathrm{~nm}$ reported in $0.050 \mathrm{~nm}$ intervals. Sutton, 2009. 5p.

LEE, K.S.; SUDDUTH, K.A.; DRUMMOND, T.S.; LEE, D.H.; KITCHEN, N.R. \& CHUNG, S.O. Calibration methods for soil property estimation using reflectance spectroscopy. Am. Soc. Agric. Biol. Eng., 53:675-684, 2010.

MONTGOMERY, O.L. \& BAUMGARDNER, M.F. The effects of the physical and chemical properties of soil on the spectral reflectance of soils. West Lafayette, Purdue University, 1974. (LARS Information Note, 112674)

MONTGOMERY, O.L.; BAUMGARDNER, M.F. \& WEISMILLER, W.A. An investigation of the relationship between spectral reflectance and chemical, physical and genetic characteristics of soils. West Lafayette, Purdue University, 1976. (LARS. Information Note, 082776)
NANNI, M.R. Dados radiométricos obtidos em laboratório e no nível orbital na caracterização e mapeamento de solos. Piracicaba, Escola superior de Agricultura Luiz de Queiroz, 2000. 366p. (Tese de Doutorado)

NANNI, M.R.; DEMATTÊ, J.A.M. \& FIORIO, P.R. Análise discriminante dos solos por meio de resposta espectral no nível terrestre. Pesq. Agropec. Bras., 39:995-1006, 2004.

NANNI, M.R. \& DEMATTÊ, J.A.M. Spectral reflectance methodology in comparison to traditional soil analysis. Soil Sci. Soc. Am. J., 70:393-407, 2006a.

NANNI, M.R. \& DEMATTÊ, J.A.M. Comportamento da linha do solo obtida por espectrorradiometria laboratorial para diferentes classes de solo. R. Bras. Ci. Solo, 30:1031-1038, 2006b.

NANNI, M.R.; CEZAR, E.; CHICATI, M.L.; HATA, F.T.; OLIVEIRA, R.B. \& DEMATTÊ, J.A. Discriminação de unidades de paisagem para fins de levantamentos pedológicos por meio da resposta espectral orbital. Acta Sci. Agron., 32:555-561, 2010.

OBUKHOV, A.I. \& ORLOV, D.S. Spectral reflectivity of the major soils group and possibility of using diffuse reflection in soil investigation. Sov. Soil Sci., 1:174-184, 1964.

PIRIE, A.; SINGH, B. \& ISLAM, K. Ultra-violet, visible, nearinfrared, and mid-infrared diffuse reflectance spectroscopic techniques to predict several soil properties. Aust. J. Soil. Res., 43:713-721, 2005.

RESENDE, M.; CURI, N.; KER, J.C. \& REZENDE, S.B. Mineralogia de solos brasileiros: Interpretação e aplicações. Lavras, Universidade Federal de Lavras, 2005. $192 p$.

SAS Institute. Statistical analyses system. Software: User's guide, version 8.2. Cary, 2001.

SOUZA, G.S. Introdução aos modelos de regressão linear e não-linear. Brasília, Embrapa/SPI/Embrapa/SEA, 1998. $505 \mathrm{p}$.

SORENSEN, L.K. \& DALSGAARD, S. Determination of clay and other soil properties by near infrared spectroscopy. Soil Sci. Soc. Am. J., 69:159-167, 2005.

SOUSA JÚNIOR, J.G.A.; DEMATTÊ, J.A.M. \& GENÚ, A.M. Comportamento espectral dos solos na paisagem a partir de dados coletados por sensores terrestre e orbital. R. Bras. Ci. Solo, 32:727-738, 2008.

SOUSA JÚNIOR, J.G.A.; DEMATTÊ, J.A.M. \& ARAÚJO, S.R. Modelos espectrais terrestres e orbitais na determinação de teores de atributos dos solos: potencial e custos. Bragantia, 70:610-621, 2011.

STENBERG, B.; JONSSON, A. \& BORJESSON, T. Near infrared technology for soil analysis with implications for precision agriculture. In: NEAR INFRARED SPECTROSCOPY: PROCEEDINGS OF THE 10TH INTERNATIONAL CONFERENCE, Chichester, 2002. Anais...Chichester, NIR Publications, 2002. p.279-284.

STENBERG, B.; VISCARRA ROSSEL, R.A.; MOUAZEN, A.M. \& WETTERLIND, J. Visible and near infrared spectroscopy in soil science. Adv. Agron., 107:164-206, 2010. 
STONER, E.R. Physicochemical, site and bi-directional reflectance factor characteristics of uniformly moist soils. West Lafayette, Purdue University, 1979. 132p. (Tese de Doutorado)

SHEPHERD, K.D. \& WALSH, M.G. Development of reflectance spectral libraries for characterization of soil properties. Soil Sci. Soc. Am. J., 66:988-998, 2002.
VISCARRA ROSSEL, R.A.; CATTLE, S.R.; ORTEGA, A. \& FOUAD, Y. In situ measurements of soil colour, mineral composition and clay content by vis-NIR spectroscopy. Geoderma, 150:253-266, 2009.

WETTERLIND, J.; STENBERG, B. \& SODERSTROM, M. Increased sample point density in farm soil mapping by local calibration of visible and near-infrared predictions models. Geoderma, 156:152-160, 2010. 